\title{
ON MODELLING RECRYSTALLIZATION PROCESSES WITH RANDOM GROWTH VELOCITIES OF THE GRAINS IN MATERIALS SCIENCE
}

\author{
ELENA VILLA ${ }^{\otimes, 1}$ AND PAULO R Rios ${ }^{2}$ \\ ${ }^{1}$ Dept. of Mathematics, University of Milan, Via Saldini 50, 20133, Milano, Italy; ${ }^{2}$ Universidade Federal \\ Fluminense, Escola de Engenharia Industrial Metalúrgica de Volta Redonda, Av. dos Trabalhadores 420, \\ 27255-125, Volta Redonda, RJ, Brasil \\ e-mail: elena.villa@unimi.it, prrios@id.uff.br \\ (Received April 24, 2012; revised July 4, 2012; accepted July 16, 2012)
}

\begin{abstract}
Heterogeneous transformations (or reactions) may be defined as those transformations in which there is a sharp moving boundary between the transformed and untransformed region. In materials science such transformations are normally called nucleation and growth transformations, whereas birth-and-growth processes is the preferred denomination in mathematics. Recently, the present authors in a series of papers have derived new analytical expressions for nucleation and growth transformations with the help of stochastic geometry methods. Those papers focused mainly on the role of nuclei location in space, described by point processes, on transformation kinetics. In this work we focus on the effect that a random velocity of the moving boundaries of the grains has in the overall kinetics. One example of a practical situation in which such a model may be useful is that of recrystallization. Juul Jensen and Godiksen reviewed recent 3D experimental results on recrystallization kinetics and concluded that there is compelling evidence that every grain has its own distinct growth rate. Motivated by this practical application we present here new general kinetics expressions for various situations of practical interest, in which a random distribution of growth velocities is assumed. In order to do this, we make use of tools from stochastic geometry and geometric measure theory. Previously known results follow here as particular cases. Although the motivation for this paper was recrystallization, the expressions derived here may be applied to nucleation and growth reactions in general.
\end{abstract}

Keywords: birth-and-growth process, casual cone, formal kinetics, phase transformations, Poisson process, random set, recrysrallization.

\section{INTRODUCTION}

Heterogeneous transformations or reactions may be defined as those transformations in which there is a sharp moving boundary between the transformed and untransformed region. This definition aims at chemical reactions in general. Specifically, it is applied to nucleation and growth transformations in materials science.

Notwithstanding its specific application to materials science, the geometrical idea pertaining to the definition of heterogeneous transformations is quite general. Indeed in mathematics this geometrical idea is called a birth and growth process. In treating heterogeneous transformations one normally envisages that these transformations may be decomposed in two stages. In the first stage, the nucleation or birth stage, new transformed regions originate at certain "nucleation sites". In the second stage, the growth stage, those nuclei, which are very small transformed regions, grow consuming the parent untransformed matrix. One might see the nucleation/birth as the stage in which the interfaces between the transformed and untransformed regions are "created".
As expected, such a general geometrical idea finds a wide range of application in diverse fields of knowledge (Tomellini and Fanfoni, 2008), such as the phase separations in multicomponent alloys (Starink, 2004), the film growth on solid substrates (Fanfoni and Tomellini, 2005), the kinetics of Ising lattice-gas model (Ramos et al., 1999), and the DNA replication (Jun and Bechhoefer, 2005). To these we may add a recent extensive work by Aquilano et al. (2009) on crystallization processes. In the present paper, the practical application that we often bear in mind is the modeling of phase transformations in general and recrystallization in particular.

For the development of mathematical models, nucleation and growth or birth and growth may be considered to be operational concepts. In other words, no specific physical mechanism is associated with nucleation and growth. They are wholly defined by their stochastic geometric properties. These ideas constitute the basis of a methodology to analyse transformation kinetics, which is often called "formal kinetics". Formal kinetics had its inception in the early work by Kolmogorov (1937), Johnson and Mehl (1939) and Avrami (1939; 1940; 
1941), henceforward referred to as "JMAK". It is worthy of note that JMAK papers were originally motivated by phase transformations. Indeed, examples of formal kinetics modeling can be found associated with a variety of transformations, such as, austenite to perlite transformation (Johnson and Mehl, 1939), recrystallization (Vandermeer and Juul Jensen, 2001), abnormal grain growth in $\mathrm{BaTiO}_{3}$ (Kondo et al., 1998), martensite "spread "(Rios and Guimaraes, 2007; 2008) and polymer crystallization (Capasso et al., 2003; Burger et al., 2002). The early work quoted above considered that nucleation sites were uniform randomly located in space, or more precisely, that nuclei were located in space according to a homogeneous Poisson point process. They also considered that the velocity of the moving boundaries was constant and was the same at every point of the moving boundaries. Based on these assumptions, two exact analytical expressions could be obtained. In both cases, one assumed that the new phase grows with a spherical shape and constant velocity, $G$, but with different nucleation conditions. In the first of these, the nucleation is supposed to be site- saturated, which means that all possible nucleation sites are exhausted at the very beginning of the reaction. One often describes this by saying that all nuclei are already present at $t=0$. If the number of nuclei per unit of volume is $N_{V}$, then the volume fraction transformed, $V_{V}$, is

$$
V_{V}(t)=1-\exp \left(-\frac{4 \pi}{3} N_{V} G^{3} t^{3}\right)
$$

whereas for a constant nucleation rate per unit of volume, $I_{V}$,

$$
V_{V}(t)=1-\exp \left(-\frac{\pi}{3} I_{V} G^{3} t^{4}\right) .
$$

Subsequent work generalized both the distribution of the nuclei in space (Rios and Villa, 2009; Villa and Rios, 2009; 2010; 2011), and the timedependence of the growth velocity (Vandermeer $e t$ al., 1991). Still, the key assumption about the velocity made in the work of JMAK remained the same; namely, that the velocity of the moving boundary at a particular point in time was the same at all points of the moving boundaries. This condition is slightly relaxed when the growth model is assumed to be shape-preserving, for instance the growing regions are ellipsoids of invariant shape (Vandermeer et al., 1991; Rios and Villa, 2009). More general growth models admitting different velocities for different boundary points can be obtained by assuming space-and-time dependent velocity $G=$ $G(t, x)$ (e.g., see Capasso et al., 2003; Burger et al., 2002; Capasso and Villa, 2007b; Villa, 2008 and references therein). In all the mentioned models the growth velocity field is assumed to be deterministic; such an assumption is possibly a good approximation for certain practical cases, whereas for others the boundary velocity may not reasonably be thought to be neither deterministic nor to be the same for each grain. As an example, suppose that spherical regions nucleate at uniform randomly located nuclei. Suppose further that their growth is controlled by the diffusion of an alloying element in the untransformed region. One concrete case would be the nucleation and growth of ferrite from austenite in an iron-carbon alloy. Ferrite has virtually no carbon and, as it grows, carbon must diffuse away from the moving ferriteaustenite interface. Eventually, carbon diffusion fields in the austenite around adjacent a growing ferrite region must overlap. This overlapping will obviously change the velocity of the moving boundaries. Because interparticle distance is random it is reasonable to infer that when overlapping starts to take place each growing ferrite region should have it is own distinct velocity. In the situation of growing ferrite just described, it is qualitatively reasonable and intuitive to expect a deviation from JMAK's velocity assumption. Unfortunately, it is not so easy to assess whether or not such an effect might be quantitatively important. One of the strong reasons for this is that measuring the boundary velocity of individually growing grains presents a significant challenge.

Juul Jensen and her group at RIS $\varnothing$ laboratories, e.g., Lauridsen et al. (2003), developed a methodology, which can non-destructively measure the growth kinetics of individual bulk grains in situ by 3-dimensional synchrotron $\mathrm{X}$-ray diffraction (3DXRD) microscopy. Juul Jensen and coworkers applied those techniques mainly to determine the growth velocity of individual grains during the recrystallization of pure aluminum. In a recent paper, Juul Jensen and Godiksen (2008) reviewed their experimental measures of growth velocities of individual grains obtained by neutron and 3dimensional synchrotron X-ray methods. They concluded that there is compelling evidence to support that "every single grain has its own kinetics different from the other grains" (Juul Jensen and Godiksen, 2008). In order to model such a situation, Juul Jensen and Godiksen emphasize that it is necessary to assume that there is a continuous distribution of growth velocities (Juul Jensen et al., 2002; Godiksen et al., 2007).

Nonetheless, in spite of this experimental evidence very few papers deal with this problem theoretically. Godiksen et al. (2007) approached this problem using computer simulation. Juul Jensen et al. (2002) derived 
an analytical expression for the volume fraction transformed for the case in which nucleation was sitesaturated.

In this paper, we obtain general analytical solutions to the nucleation and growth model considering that there is a probability distribution of growth velocities of the grains. We published a preliminary version of this work (Rios and Villa, 2011), in which we focused on particular cases of practical interest. In this paper we present a more detailed theoretical mathematical treatment covering several and more general cases. Namely, we consider the cases in which the velocity of each grain is

- random but constant in time

- random and time-dependent

- random dependent on the spatial location of the corresponding nucleus,

both in the case of site-saturation and in the case of time dependent nucleation. It is worthy of note that even though we have been motivated by the observations of recrystallization behavior (Juul Jensen and Godiksen, 2008) the present results may be applied quite generally to birth-and-growth processes. Also for this reason, throughout the paper we provide explicit expressions for arbitrarily integer dimension $d$, so that the 2D and 3D case usually considered in materials science applications follow as particular cases.

\section{MATHEMATICAL BACKGROUND AND BASIC NOTATION}

Detailed mathematical background may be found in previous work by the authors Rios and Villa (2009) and Capasso and Villa (2007a;b); in particular we refer to our recent paper (Villa and Rios, 2011) for basic definitions. Here only some essential definitions and some useful relationships will be presented to make this paper more self contained and easier to read. For a brief description of homogeneous and inhomogeneous Poisson point process, the reader is referred to Rios and Villa (2009) or for a more detailed presentation to specific texts on stochastic geometry (Stoyan et al., 1995).

\section{MODELLING BIRTH-AND-GROWTH PROCESSES}

A birth-and-growth (stochastic) process is a dynamic germ-grain model (Stoyan et al., 1995), used to model situations in which nuclei (germs) are born in time and are located in space randomly, and each nucleus generates a grain evolving in time according with a given growth law. Since, in general, nucleation and growth are random in time and space, then the transformed region $\Theta^{t}$ at any time $t>0$ is a random set (Stoyan et al., 1995) in $\mathbb{R}^{d}$, that is a measurable map from a probability space $(\Omega, \mathfrak{F}, \mathbb{P})$ to the space of closed subsets in $\mathbb{R}^{d}$; namely

$$
\Theta^{t}:(\Omega, \mathfrak{F}, \mathbb{P}) \longrightarrow\left(\mathbb{F}, \sigma_{\mathbb{F}}\right),
$$

where $\mathbb{F}$ denotes the class of the closed subsets in $\mathbb{R}^{d}$, and $\sigma_{\mathbb{F}}$ is the $\sigma$-algebra generated by the so called Fell topology, or hit-or-miss topology, that is the topology generated by the set system

$$
\left\{\mathscr{F}_{G}: G \in \mathscr{G}\right\} \cup\left\{\mathscr{F}^{C}: C \in \mathscr{C}\right\},
$$

where $\mathscr{G}$ and $\mathscr{C}$ are the system of the open and compact subsets of $\mathbb{R}^{d}$, respectively (e.g., see Matheron, 1975; Schneider and Weil, 2008). The family $\left\{\Theta^{t}\right\}_{t}$ is called birth-and-growth process. Birth-and-growth and nucleation and growth will be used as synonyms in this paper. Of course, different kinds of nucleation and growth models gives rise to different kinds of processes $\left\{\Theta^{t}\right\}_{t}$. As mentioned in the Introduction, we shall consider two kinds of nucleation of interest in recrystallization; the first one is that of sitesaturation, in which all nucleation sites are exhausted at the beginning of the reaction, the second one is that of time-dependent nucleation, in which nuclei can be born randomly both in space and time. Site-saturated nucleation processes and space-time dependent nucleation processes can be modeled by point processes and marked point processes, respectively. We give here some basic concepts and definitions useful for the sequel. (See also, for instance, Rios and Villa, 2009, Sec. 2.3.) Throughout the paper $\mathscr{B}_{\mathscr{X}}$ denotes the Borel $\sigma$-algebra of any space $\mathscr{X}$. We remind that a point process in $\mathbb{R}^{d}$ is an almost surely locally finite sequence of points, say $N=$ $\left\{X_{i}\right\}_{i}$, randomly located in $\mathbb{R}^{d}$, according with a given probability law. Equivalently, it can be seen also as a counting measure as well, so defined

$$
N(A):=\text { number of the } X_{i} \text { 's, which belong to } A,
$$

for any $A \in \mathscr{B}_{\mathbb{R}^{d}}$. Therefore a point process $N$ can be interpreted both as a random sequence of points and as a counting measure; this is the reason why the same notation $N$ is commonly used. Then, denoted by $X_{i}$ the spatial location of the $j$-th nucleus of a site-saturated nucleation process, and by $\Theta^{t}\left(X_{j}\right)$ the grain obtained as the evolution up to time $t>0$ of the nucleus $X_{j}$, the transformed region $\Theta^{t}$ at time $t>0$ is

$$
\Theta^{t}=\bigcup_{j} \Theta^{t}\left(X_{j}\right) .
$$


Given a complete separable metric space $\mathbf{K}$, a marked point process in $\mathbb{R}_{+} \times \mathbf{K}$ is a sequence $N:=\left\{\left(T_{i}, K_{i}\right)\right\}_{i}$ of points in $\mathbb{R}_{+} \times \mathbf{K}$ such that the sequence $\left\{T_{i}\right\}_{i}$ is a point process in $\mathbb{R}_{+}$, while each $K_{i} \in \mathbf{K}$ is said to be the mark associated to the point $T_{i}$. $\mathbf{K}$ is called mark space. Then, a space-time nucleation process can be modeled by a marked point process, identifying $T_{i}$ as the time of birth of the $i$-th nucleus, and $X_{i}$ as its spatial location in $\mathbb{R}^{d}$. Analogously to the site-saturation case, a counting process $N$ on $\mathbb{R}_{+} \times \mathbb{R}^{d}$ can be defined as

$$
\begin{aligned}
N\left([s, t] \times A^{\prime}\right):= & \text { number of nuclei, which are born } \\
& \text { in } A^{\prime} \text { during the time interval }[s, t] .
\end{aligned}
$$

By denoting $\Theta_{T_{j}}^{t}\left(X_{j}\right)$ the grain obtained as the evolution up to time $t \geq T_{j}$ of the nucleus born at time $T_{j}$ in $X_{j}$, then the transformed region $\Theta^{t}$ at time $t>0$ is given by

$$
\Theta^{t}=\bigcup_{T_{j} \leq t} \Theta_{T_{j}}^{t}\left(X_{j}\right)
$$

Of course a site saturated process may be seen as a particular case of the time-dependent one by assuming $T_{j} \equiv 0$ for any $j$.

Given a point process $N$ on $\mathbb{R}^{d}$, the measure $\Lambda(A):=\mathbb{E}[N(A)]$ for all $A \in \mathscr{B}_{\mathbb{R}^{d}}$ is called intensity measure of $N$; whereas, if $N$ is a marked point process process in $\mathbb{R}_{+} \times \mathbf{K}$, then its intensity measure $\Lambda$ is the measure on $\mathbb{R}_{+} \times \mathbf{K}$ defined as $\Lambda(A):=\mathbb{E}[N(A)]$ for all $A \in \mathscr{B}_{\mathbb{R}_{+}} \times \mathscr{B}_{\mathbf{K}}$. In other words, if $N$ is a sitesaturated nucleation process, then $\Lambda(A)$ represents the mean number of nuclei born in $A \subset \mathbb{R}^{d}$; whereas if $N=\left(T_{j}, X_{j}\right)_{j}$ is a time-dependent nucleation process, then $\Lambda\left([s, t] \times A^{\prime}\right)$ is the mean number of nuclei born in $A^{\prime}$ during a time interval $[s, t]$. If the marks are independent and identically distributed, and independent of the unmarked point process $\left\{T_{i}\right\}_{i}$, then the common probability distribution, say $Q$, of the marks is called mark distribution, the process $N$ is said to be an independent marking of $\left\{T_{i}\right\}_{i}$, and $\Lambda$ is of the type $\Lambda(\mathrm{d}(s, y))=\lambda(\mathrm{d} s) Q(\mathrm{~d} y)$. We mention that the notion of marked point process will be useful in the sequel for modelling also birth-and-growth processes whose nucleation process is site-saturated and the growth velocity of each grain is a random quantity, which can be described as a mark associated to the corresponding nucleus. In this paper we shall assume Poissonian nucleation, that is $N$ will be a (marked) Poisson process.

In order to define a birth-and-growth process we need to introduce also a growth model. Models of volume growth have been studied extensively, since the pioneering work by Kolmogorov (1937). We consider here a simple case of the so-called normal growth model (see also, e.g., Capasso and Villa, 2007b; Villa, $2010 \mathrm{~b}$ and references therein); namely, we shall consider the case in which all the grains develop with random velocity $G$ constant in time or time dependent, so that for any time $t$ all the grains have spherical shape (this is due to the fact that $G$ is not space-dependent).

\section{MEAN DENSITIES ASSOCIATED TO A BIRTH-AND-GROWTH PROCESS}

Since $\Theta^{t}$ is a random set, it gives rise to a random measure $v^{d}\left(\Theta^{t} \cap \cdot\right)$ in $\mathbb{R}^{d}$ for all $t>0$, having denoted by $v^{d}$ the $d$-dimensional Lebesgue measure in $\mathbb{R}^{d}$. In particular, it is of interest to consider the expected volume measure $\mathbb{E}\left[v^{d}\left(\Theta^{t} \cap \cdot\right)\right]$ and its density (i.e., its Radon-Nikodym derivative), called mean volume density of $\Theta^{t}$ and denoted by $V_{V}$, provided it exists:

$$
\mathbb{E}\left[v^{d}\left(\Theta^{t} \cap A\right)\right]=\int_{A} V_{V}(t, x) \mathrm{d} x, \quad \forall A \in \mathscr{B}_{\mathbb{R}^{d}} .
$$

It is well known and easy to prove that

$$
V_{V}(t, x)=\mathbb{P}\left(x \in \Theta^{t}\right), \quad \text { for } v^{d} \text {-a.e. } x \in \mathbb{R}^{d} .
$$

Whenever $A$ is the region of the physical sample under observation, the ratio

$$
\mathbf{V}_{\mathbf{V}}(t, A):=\frac{\int_{A} V_{V}(t, x) \mathrm{d} x}{V^{d}(A)}
$$

is also called volume fraction. Let us notice that whenever $V_{V}$ is independent of $x$ (e.g., under assumptions of homogeneous nucleation and growth), then $\mathbf{V}_{\mathbf{V}}$ is independent of $A$ and $\mathbf{V}_{\mathbf{V}}(t)=V_{V}(t)$ (Rios and Villa, 2009; Stoyan et al., 1995, p. 342). We also mention that other quantities of interest in real applications are the so-called mean extended volume density at time $t$, denoted by $V_{E}(t, \cdot)$, defined as the density of the mean extended volume measure at time $t, \mathbb{E}\left[\mu_{\Theta^{t}}^{\mathrm{ex}}\right](\cdot):=\mathbb{E}\left[\sum_{j: T_{j} \leq t} \nu^{d}\left(\Theta_{T_{j}}^{t}\left(X_{j}\right) \cap \cdot\right)\right]$ on $\mathbb{R}^{d}$, that is

$$
\mathbb{E}\left[\mu_{\Theta^{t}}^{\mathrm{ex}}\right](A)=\int_{A} V_{E}(t, x) \mathrm{d} x, \quad \forall A \in \mathscr{B}_{\mathbb{R}^{d}},
$$

and the mean surface density $S_{V}(t, \cdot)$ and the mean extended surface density $S_{E}(t, \cdot)$ at time $t$, defined as the density of the mean surface measure at time $t, \mathbb{E}\left[\mu_{\partial \Theta^{t}}\right](\cdot):=\mathbb{E}\left[\mathscr{H}^{d-1}(\partial \Theta \cap \cdot)\right]$ and of the mean extended surface measure at time $t, \mathbb{E}\left[\mu_{\partial \Theta^{t}}^{e x}\right](\cdot):=$ $\mathbb{E}\left[\sum_{j: T_{j} \leq t} \mathscr{H}^{d-1}\left(\partial \Theta_{T_{j}}^{t}\left(X_{j}\right) \cap \cdot\right)\right]$, respectively, where $\mathscr{H}^{d-1}$ is the $(d-1)$-dimensional Hausdorff measure. In other words, the mean extended volume and surface measures represent the mean of the sum of the volume measures and of the surface measures of the grains which are born and grown until time $t$, supposed free to grow, ignoring overlapping (Rios and Villa, 2009; 
Villa, 2008). It is clear that to find out formulas for the mean volume density $V_{V}$ (and so for $\mathbf{V}_{\mathbf{V}}$ and the other quantities we mentioned above, as a consequence) is of particular interest in real applications.

\section{CAUSAL CONE}

The causal cone notion plays, together with Eq. 2, a fundamental role in evaluating the mean volume density $V_{V}(t, x)$ of $\Theta^{t}$ at a point $x$. We recall the following definition (Kolmogorov, 1937):

Definition 1 (Causal cone) If $N$ is a nucleation process on a suitable space $\mathscr{X}$, then the causal cone $\mathscr{C}(t, x)$ of a point $x$ at time $t$ is the subset of $\mathscr{X}$ in which at least one nucleation event has to take place in order to cover the point $x$ at time $t$.

Explicit expressions for the causal cone will be provided throughout the paper in relation with the different kinds of birth-and-growth processes we are going to consider. In order to give a first more intuitive notion of the causal cone, let us consider the very particular case of site-saturation with constant growth velocity of the grains $G$; in this case, a point $x$ will be transformed at time $t$ if at least one nucleus is born in the ball with radius $R=G t$ centred in $x$, therefore such a ball is the causal cone $\mathscr{C}(t, x)$ of the point $x$ at time $t$ in this case.

\section{PARTICULAR CASE: DETERMINISTIC SPACE-INDEPENDENT VELOCITY}

We briefly recall the well known results for the mean densities of a birth-and-growth process $\Theta^{t}$ with normal growth model in the case in which the velocity of each grain is assumed to be deterministic and spaceindependent. Throughout the paper $B_{r}(x)$ denotes the ball centred in $x$ with radius $r$. So, let $N$ be the nucleation process with intensity measure $\Lambda$, and $G=$ $G(t)$ be the growth velocity of each grain at any time $t$; then it is clear that at any time $t$ each grain has spherical shape, namely

$$
\Theta^{t}\left(X_{j}\right)=B_{R(t)}\left(X_{j}\right), \quad R(t)=\int_{0}^{t} G(\tau) \mathrm{d} \tau,
$$

in the site-saturated nucleation case, and

$$
\Theta_{T_{j}}^{t}\left(X_{j}\right)=B_{R\left(T_{j}, t\right)}\left(X_{j}\right), \quad R\left(T_{j}, t\right)=\int_{T_{j}}^{t} G(\tau) \mathrm{d} \tau,
$$

in the time-dependent nucleation case. As a consequence, by Eq. 2 and the definition of the causal cone, it follows that

$$
V_{V}(t, x)=\mathbb{P}(N(\mathscr{C}(t, x))>0),
$$

where

$$
\mathscr{C}(t, x):=\left\{y \in \mathbb{R}^{d}: x \in B_{R(t)}(y)\right\}=B_{R(t)}(x)
$$

in the site-saturated case, and

$$
\begin{aligned}
\mathscr{C}(t, x) & :=\left\{(s, y) \in[0, t] \times \mathbb{R}^{d}: x \in B_{R(s, t)}(y)\right\} \\
& =\left\{(s, y) \in[0, t] \times \mathbb{R}^{d}: y \in B_{R(s, t)}(x)\right\}
\end{aligned}
$$

in the time-dependent case (Rios and Villa, 2009, Sec 2.4).

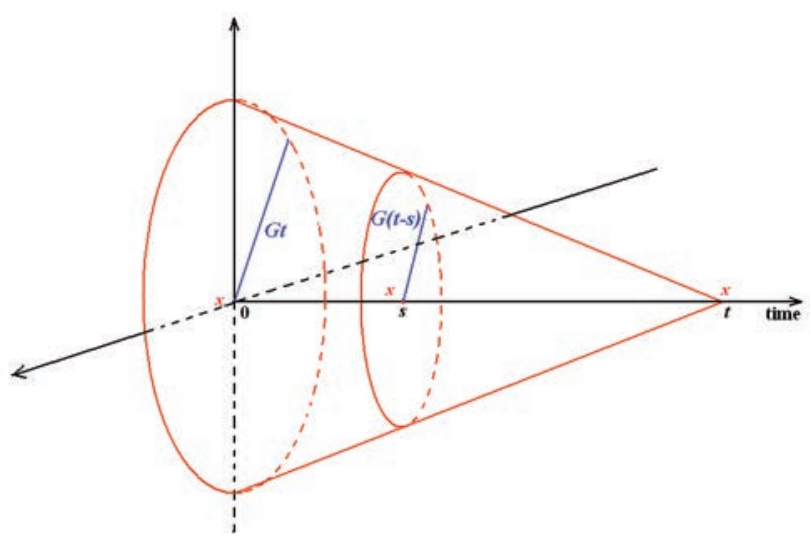

Fig. 1. A geometric interpretation of the causal cone $\mathscr{C}(t, x)$ of a point $x \in \mathbb{R}^{d}$ at time $t$, in the timedependent nucleation case, assuming $G$ constant. The vertical and horizontal axis represent the space $\mathbb{R}^{d}$ and the time, respectively. Note that for any $s \in[0, t]$, the section of the causal cone is the set of points in $\mathbb{R}^{d}$ where a nucleation event has to take place in order to cover the point $x$ at time $t$; so, in this case, it is the ball centred in $x$ with radius $G(t-s)$.

Moreover, we recall that (Villa, 2008)

$$
V_{E}(t, x)=\Lambda(\mathscr{C}(t, x)),
$$

and that

$$
G(t)=\frac{1}{S_{V}(t, x)} \frac{\partial V_{V}(t, x)}{\partial t}=\frac{1}{S_{E}(t, x)} \frac{\partial V_{E}(t, x)}{\partial t},
$$

finally, under Poissonian assumption on the nucleation process it holds (Schneider and Weil, 2008, Eq. 11.12, and references therein),

$$
V_{V}(t, x)=1-\mathrm{e}^{-V_{E}(t, x)},
$$

and

$$
S_{V}(t, x)=\left(1-V_{V}(t, x)\right) S_{E}(t, x) .
$$




\section{RANDOM VELOCITY IN THE SITE- SATURATED CASE}

In this section we consider the case in which all nucleation sites are exhausted at the beginning of the reaction (site-saturation) and the growth velocity of the grains is random; in particular we consider three different cases of interest:

1. the velocity $G_{i}$ associated to the grain with nucleus $X_{i}$ is constant during the reaction, but random;

2. the velocity $G_{i}$ associated to the grain with nucleus $X_{i}$ is random and time dependent;

3. the velocity $G_{i}$ associated to the grain with nucleus $X_{i}$ is constant during the reaction, but random with probability distribution dependent on the specific location of the nucleus.

In all of the three above mentioned cases, we assume that the point process $\left\{X_{j}\right\}_{j}$ describing the spatial location of the nuclei is an inhomogeneous Poisson point process in $\mathbb{R}^{d}$ with intensity $\lambda(x)$. Our aim is to find out general results for the mean densities of the transformed region $\Theta^{t}$, reobtaining the known above mentioned results (Eqs. 4, 5, 6 and 7) when the velocity is not random, as particular case.

\section{RANDOM CONSTANT VELOCITY}

Assumptions: each grain develops with random velocity constant in time during the reaction; different grains may have different velocity. The velocity $G_{i}$ associated to the grain with nucleus $X_{i}$ has a probability distribution $Q$, independent on the spatial location of the nucleus, such that $\mathbb{E}\left[G^{d}\right]<\infty$.

Therefore we may consider the velocity associated to the nucleus $X_{i}$ as a mark of the point $X_{i}$, and model such a birth-and-growth process by a marked Poisson point process $N=\left\{X_{i}, G_{i}\right\}_{i}$ in $\mathbb{R}^{d}$ with pairwise independent marks in $\mathbb{R}_{+}$, with mark distribution $Q$. Thus the intensity measure $\Lambda$ of the nucleation process $N$ is of the type

$$
\Lambda(\mathrm{d}(x, \xi))=\lambda(x) \mathrm{d} x Q(\mathrm{~d} \xi),
$$

while the transformed region $\Theta^{t}$ at time $t$ is given by

$$
\Theta^{t}=\bigcup_{\left(X_{i}, G_{i}\right) \in \Phi} B_{G_{i} t}\left(X_{i}\right) .
$$

We start to prove that Eq. 4 and Eq. 6 hold also in this setting. Let us notice that, by definition, the causal cone $\mathscr{C}(t, x)$ of a point $x \in \mathbb{R}^{d}$ at time $t$ will be now the set of all the pairs $(y, \xi) \in \mathbb{R}^{d} \times \mathbb{R}_{+}$, such that a grain born at point $y$ that grows with velocity $\xi$ covers the point $x$ at time $t$; it is worthy of note that in contrast with the causal cone shown in Fig. 1, the visualization of such a causal cone is difficult.

The results stated in the next theorem can be easily derived by the well known theory for inhomogeneous Boolean models with spherical grains (Fallert, 1996; Schneider and Weil, 2008); we give here a sketch of the proof for a non-expert reader's convenience.

Theorem 2 Under the above assumptions, Eqs. 4 and 6 hold with

$$
\mathscr{C}(t, x)=\left\{(y, \xi) \in \mathbb{R}^{d} \times \mathbb{R}_{+}: y \in B_{\xi}(x)\right\} .
$$

Sketch of the proof. By definition

$$
\mathscr{C}(t, x):=\left\{(y, \xi) \in \mathbb{R}^{d} \times \mathbb{R}_{+}: x \in B_{\xi t}(y)\right\}=\text { Eq. } 9,
$$

and so, being $N$ a Poisson process, it follows that

$$
\begin{aligned}
V_{V}(t, x) & =1-\exp \{-\Lambda(\mathscr{C}(t, x))\} \\
& =1-\exp \left\{-\int_{\mathbb{R}_{+}} \int_{B_{\xi_{t}}(x)} \lambda(y) \mathrm{d} y Q(\mathrm{~d} \xi)\right\} .
\end{aligned}
$$

By the definition (Eq. 3) of $V_{E}$, and by the application of the Campbell's formula (Baddeley et al., 2007),

$$
\left.\int_{A} V_{E}(t, x) \mathrm{d} x=\int_{\mathbb{R}_{+}} \int_{\mathbb{R}^{d}} v^{d}\left(B_{\xi t}(y) \cap A\right)\right) \lambda(y) \mathrm{d} y Q(\mathrm{~d} \xi)
$$

for any $A \in \mathscr{B}_{\mathbb{R}^{d}}$. By applying Fubini's theorem and a suitable change of variable, we get

$$
\begin{aligned}
& \int_{A} V_{E}(t, x) \mathrm{d} x \\
& =\int_{\mathbb{R}_{+}} \int_{\mathbb{R}^{d}} \lambda(y)\left(\int_{\mathbb{R}^{d}} \mathbf{1}_{B_{\xi t}(y)}(x) \mathbf{1}_{A}(x) \mathrm{d} x\right) \mathrm{d} y Q(\mathrm{~d} \xi) \\
& \stackrel{\text { Eq. }}{=} \int_{A} \Lambda(\mathscr{C}(t, x)) \mathrm{d} x
\end{aligned}
$$

for any $A \in \mathscr{B}_{\mathbb{R}^{d}}$, and so the assertion.

Similarly, we may find out an explicit formula for the mean extended surface density $S_{E}$.

Theorem 3 Under the above assumptions, it follows that

$$
S_{E}(t, x)=\int_{\mathbb{R}_{+}} \int_{\partial B_{\xi_{t}}(z)} \lambda(y) \mathscr{H}^{d-1}(\mathrm{~d} y) Q(\mathrm{~d} \xi) .
$$


Proof. By proceeding along the same lines of the previous theorem, by the definition of $S_{E}$, and by the application of the Campbell's formula, the following chain of equalities holds for any $A \in \mathscr{B}_{\mathbb{R}^{d}}$ :

$$
\begin{aligned}
\int_{A} & S_{E}(t, x) \mathrm{d} x \\
:= & \mathbb{E}\left[\sum_{\left(X_{i}, G_{i}\right) \in N} \mathscr{H}^{d-1}\left(\partial B_{G_{i} t}\left(X_{i}\right) \cap A\right)\right] \\
= & \left.\int_{\mathbb{R}_{+}} \int_{\mathbb{R}^{d}} \mathscr{H}^{d-1}\left(\partial B_{\xi t}(y) \cap A\right)\right) \lambda(y) \mathrm{d} y Q(\mathrm{~d} \xi) \\
= & \int_{\mathbb{R}_{+}} \int_{\mathbb{R}^{d}} \lambda(y) \\
& \left(\int_{\mathbb{R}^{d}} \mathbf{1}_{\partial B_{\xi_{t}}(y)}(x) \mathbf{1}_{A}(x) \mathscr{H}^{d-1}(\mathrm{~d} x)\right) \mathrm{d} y Q(\mathrm{~d} \xi) \\
= & \int_{\mathbb{R}_{+}} \int_{\mathbb{R}^{d}} \int_{\mathbb{R}^{d}} \lambda(z-s) \\
& \mathbf{1}_{\partial B_{\xi_{t}}(0)}(s) \mathbf{1}_{A}(z) \mathscr{H}^{d-1}(\mathrm{~d} s) \mathrm{d} z Q(\mathrm{~d} \xi) \\
= & \int_{A}\left(\int_{\mathbb{R}_{+}} \int_{\partial B_{\xi_{t}}(z)} \lambda(w) \mathscr{H}^{d-1}(\mathrm{~d} w) Q(\mathrm{~d} \xi)\right) \mathrm{d} z
\end{aligned}
$$

and so the assertion.

We want now to provide a result about the overall velocity $\mathscr{G}:=\frac{1}{S_{V}(t, x)} \frac{\partial}{\partial t} V_{V}(t, x)$ of the process; we expect to reobtain $\mathscr{G}=G>0$ in the particular case in which $G$ is deterministic, according with Eq. 5.

We recall that a function $f: \mathbb{R}^{d} \rightarrow \mathbb{R}$ is said to be harmonic if it is twice continuously differentiable and it satisfies the Laplace's equation $\sum_{i=1}^{d} \partial^{2} f / \partial x_{i}^{2}=0$. Note that, in particular, if the nucleation process is homogeneous in space, then $\lambda(x) \equiv \lambda>0$ is harmonic.

Corollary 4 Under the above assumptions, if $\lambda$ is a non-negative harmonic function in the spatial region where the nucleation takes place, then the Eqs. 4 and 11 simplify as follows:

$$
\begin{aligned}
& V_{E}(t, x)=\lambda(x) b_{d} t^{d} \mathbb{E}\left[G^{d}\right], \\
& S_{E}(t, x)=\lambda(x) d b_{d} t^{d-1} \mathbb{E}\left[G^{d-1}\right],
\end{aligned}
$$

where $b_{d}$ is the volume of the unit ball in $\mathbb{R}^{d}$. Moreover,

$$
\frac{\partial}{\partial t} V_{E}(t, x)=\frac{\mathbb{E}\left[G^{d}\right]}{\mathbb{E}\left[G^{d-1}\right]} S_{E}(t, x) .
$$

Proof. In order to obtain Eqs. 12 and 13, it is sufficient to remind that for any harmonic function $f$ in $\mathbb{R}^{d}$ it holds

$$
\int_{B_{R}(x)} f(y) \mathrm{d} y=f(x) v^{d}\left(B_{R}(0)\right)=f(x) b_{d} R^{d}, \quad \forall R \geq 0,
$$

and

$$
\int_{\partial B_{R}(x)} f(y) \mathscr{H}^{d-1}(\mathrm{~d} y)=f(x) d b_{d} R^{d-1}, \quad \forall R \geq 0,
$$

and then to apply this to the expressions for $V_{E}$ and $S_{V}$ given in the theorems above. Eq. 14 directly follows by Eqs. 12 and 13.

For sake of simplicity, we assume in the next theorem that the intensity $\lambda$ of the point process driving the spatial location of the nuclei is bounded and continuous; such an assumption is usually satisfied in real applications. We point out that the validity of Eq. 7 stated in the theorem below, may be derived by the result proved in Schneider and Weil (2008, Theorem 11.1.3); nevertheless, the proof provided there requires notions (e.g., specific intrinsic volumes and mixed measures) which might be difficult to understand for a non-expert reader. So we give here a sketch of an alternative proof, which might be appear a bit more intuitive.

Theorem 5 Under the above assumptions, if the intensity $\lambda$ is bounded and continuous, Eq. 7 still holds; moreover if $\lambda$ is harmonic in the spatial region where the nucleation takes place, then it holds

$$
\frac{\partial}{\partial t} V_{V}(t, x)=\frac{\mathbb{E}\left[G^{d}\right]}{\mathbb{E}\left[G^{d-1}\right]} S_{V}(t, x) .
$$

Sketch of the proof. By the general results on the mean density of inhomogeneous Boolean models proved in Villa (2010a) (in particular by applying here Theorem 3.9, observing that the grains of the transformed region $\Theta^{t}$ are balls for any $t>0$, and so the $\mathscr{H}^{d-1}$-measure of the topological boundary of $\Theta^{t}$ equals the $\mathscr{H}^{d-1}$-measure of its the essential boundary), we can claim that

$$
S_{V}(t, x)=\lim _{r \downarrow 0} \frac{\mathbb{P}\left(x \in \Theta_{\oplus r}^{t} \backslash \Theta^{t}\right)}{r},
$$

where $\Theta_{\oplus r}^{t}:=\left\{x \in \mathbb{R}^{d}: \operatorname{dist}\left(x, \Theta^{t}\right) \leq r\right\}$ is the parallel set of $\Theta^{t}$ at distance $r$. Let us notice that

$$
\Theta_{\oplus r}^{t}=\bigcup_{\left(X_{i}, G_{i}\right) \in N} B_{G_{i} t+r}\left(X_{i}\right)
$$

and denote by $\mathscr{D}_{r}$ the subset of $\mathbb{R}^{d} \times \mathbb{R}_{+}$so defined:

$$
\mathscr{D}_{r}:=\left\{(y, \xi) \in \mathbb{R}^{d} \times \mathbb{R}_{+}: x \in B_{\xi_{t+r}}(y)\right\} .
$$


We may observe that

$$
\begin{aligned}
& \mathbb{P}\left(x \in \Theta_{\oplus r}^{t} \backslash \Theta^{t}\right) \\
& =\mathbb{P}\left(\left\{N\left(\mathscr{D}_{r}\right)>0\right\} \cap\{N(\mathscr{C}(t, x))=0\}\right) \\
& =\mathbb{P}\left(N\left(\left\{(y, \xi): y \in B_{\xi t+r}(x) \backslash B_{\xi t}(x)\right\}\right)>0\right) \\
& \cdot \mathbb{P}(N(\mathscr{C}(t, x))=0) \\
& =\left(1-\exp \left\{-\int_{\mathbb{R}_{+}} \int_{B_{\xi t+r}(x) \backslash B_{\xi t}(x)} \lambda(y) \mathrm{d} y Q(\mathrm{~d} \xi)\right\}\right) \\
& \cdot\left(1-V_{V}(t, x)\right),
\end{aligned}
$$

where the last two equations follow by the assumption the $N$ is a Poisson point process. By applying Villa (2010a, Theorem 3.5b) to the measure $\mu(\mathrm{d} x):=$ $\lambda(x) \mathrm{d} x$, we get that

$$
\begin{aligned}
\lim _{r \downarrow 0} \frac{1}{r} \int_{B_{\xi t+r}(x) \backslash B_{\xi t}(x)} \lambda(y) \mathrm{d} y \\
=\int_{\partial B_{\xi t}(x)} \lambda(y) \mathscr{H}^{d-1}(\mathrm{~d} y),
\end{aligned}
$$

by denoting now $\bar{\lambda}:=\max _{x \in \mathbb{R}^{d}} \lambda(x)$, we observe that $\frac{1}{r} \int_{B_{\xi_{t+r}}(x) \backslash B_{\xi t}(x)} \lambda(y) \mathrm{d} y \leq \bar{\lambda} b_{d} d\left(\xi_{t}+1\right)^{d-1}, \quad \forall r \leq 1$, and

$$
\int_{\mathbb{R}_{+}} \bar{\lambda} b_{d} d(\xi t+1)^{d-1} Q(\mathrm{~d} \xi)<\infty,
$$

being $\mathbb{E}\left[G^{d}\right]<\infty$ by assumption. Therefore, by applying the dominated convergence theorem, we can exchange limit and integral in Eq. 19, and so we get

$$
\begin{aligned}
\lim _{r \rightarrow 0} \frac{1-\exp \left\{-\int_{\mathbb{R}_{+}} \int_{B_{\xi t+r}(x) \backslash B_{\xi t}(x)} \lambda(y) \mathrm{d} y Q(\mathrm{~d} \xi)\right\}}{r} \\
=\lim _{r \rightarrow 0} \frac{\int_{\mathbb{R}_{+}} \int_{B_{\xi t+r}(x) \backslash B_{\xi t}(x)} \lambda(y) \mathrm{d} y Q(\mathrm{~d} \xi)}{r} \\
=\int_{\mathbb{R}_{+}} \int_{\partial B_{\xi_{t}}(x)} \lambda(y) \mathscr{H}^{d-1}(\mathrm{~d} y) Q(\mathrm{~d} \xi) \stackrel{\text { Eq. }{ }^{11}}{=} S_{E}(t, x) .
\end{aligned}
$$

By Eqs. 16, 17 and 20 we obtain Eq.7. Finally, Eq.15 can be obtained as follows:

$$
\begin{aligned}
& \frac{\partial}{\partial t} V_{V}(t, x) \stackrel{\text { Eq. } 6}{=}\left(1-V_{V}(t, x)\right) \frac{\partial}{\partial t} V_{E}(t, x) \\
& \stackrel{\text { Eq. } 14}{=}\left(1-V_{V}(t, x)\right) \frac{\mathbb{E}\left[G^{d}\right]}{\mathbb{E}\left[G^{d-1}\right]} S_{E}(t, x) \\
& \stackrel{\text { Eq. } 7}{=} \frac{\mathbb{E}\left[G^{d}\right]}{\mathbb{E}\left[G^{d-1}\right]} S_{V}(t, x) .
\end{aligned}
$$

Therefore, in the case the intensity $\lambda$ is harmonic, the overall velocity $\mathscr{G}$ is given by $\mathbb{E}\left[G^{d}\right] / \mathbb{E}\left[G^{d-1}\right]$; moreover we note that Eqs. 14 and 15 may be seen as a generalization of Eq. 5 in the case of time-independent velocity, because in the particular case of deterministic velocity $\mathbb{E}\left[G^{d}\right] / \mathbb{E}\left[G^{d-1}\right]=G$.

Remark 6 By assumption, $G$ is a nonnegative random variable with probability distribution $Q$, and so $\mathbb{E}\left[G^{d}\right]=\int_{\mathbb{R}_{+}} \xi^{d} Q(\mathrm{~d} \xi)$, whereas $\mathbb{E}\left[G^{d-1}\right]=$ $\int_{\mathbb{R}_{+}} \xi^{d-1} Q(\mathrm{~d} \xi)$. Therefore, if we denote by $\widetilde{G}$ the nonnegative random variable with probability distribution

$$
g(\xi) \mathrm{d} \xi:=\frac{\xi^{d-1} Q(\mathrm{~d} \xi)}{\int_{\mathbb{R}_{+}} \xi^{d-1} Q(\mathrm{~d} \xi)}
$$

then $\mathbb{E}[\widetilde{G}]=\mathbb{E}\left[G^{d}\right] / \mathbb{E}\left[G^{d-1}\right]$, so that Eqs. 14 and 15 can be written in terms of $\widetilde{G}$ as follows

$$
\mathbb{E}[\widetilde{G}]=\frac{1}{S_{E}(t, x)} \frac{\partial}{\partial t} V_{E}(t, x)=\frac{1}{S_{V}(t, x)} \frac{\partial}{\partial t} V_{V}(t, x),
$$

and so the overall velocity is given by $\mathscr{G}=\mathbb{E}[\widetilde{G}]$.

Example Let the assumptions of Theorem 2 be satisfied, with $\lambda$ harmonic in the spatial region where the nucleation takes place. Then by 6 and 12 we get the following explicit expressions for the mean volume density $V_{V}(t, x)$ in a few cases of interest for applications:

- if $G$ is uniformly distributed in $[0, K]$, then

$$
V_{V}(t, x)=1-\exp \left\{-\frac{\lambda(x) b_{d}}{d+1} K^{d} t^{d}\right\}
$$

- if $G$ is Exponential with expected value $c>0$ (i.e., $\left.Q(\mathrm{~d} \xi)=\frac{1}{c} \mathrm{e}^{-\xi / c} \mathrm{~d} \xi\right)$, then

$$
V_{V}(t, x)=1-\exp \left\{-\lambda(x) b_{d} t^{d} c^{d} d !\right\}
$$

- if $G$ is a discrete random variable with $\mathbb{P}(G=$ $\left.G_{i}\right)=p_{i}, p_{i} \in[0,1], \sum_{i} p_{i}=1$, then

$$
V_{V}(t, x)=1-\exp \left\{-\lambda(x) b_{d} t^{d} \sum_{i} G_{i}^{d} p_{i}\right\} .
$$

Explicit expressions for the other mean densities $S_{V}, V_{E}$ and $S_{E}$ can be obtained similarly. 


\section{RANDOM TIME-DEPENDENT VELOCITY}

Assumptions: each grain develops with random time-dependent velocity during the reaction, of the type:

$$
G(t)=G_{0} g(t, \alpha),
$$

where $G_{0}$ is a non-negative random variable and $g$ is a non-negative function depending on time and on a random vector parameter $\alpha$ in $\mathbb{R}^{n}$. We assume that $G_{0}$ and $\alpha$ are independent on the spatial location of the nucleus of the associated grain, with joint probability distribution $Q(\mathrm{~d}(\xi, a))$ on $\mathbb{R}_{+} \times \mathbb{R}^{n}$.

Even in this case, different grains may have different velocity, and we may model such a birthand-growth process by a marked Poisson point process $N=\left\{X_{i},\left(G_{i}, \alpha_{i}\right)\right\}_{i}$ in $\mathbb{R}^{d}$ with independent marking in $\mathbb{R}_{+} \times \mathbb{R}^{n}$, with mark distribution $Q$. Then, the intensity measure $\Lambda$ of the nucleation process $N$ is of the type

$$
\Lambda(\mathrm{d}(y, \xi, a))=\lambda(y) \mathrm{d} y Q(\mathrm{~d}(\xi, a))
$$

while the transformed region $\Theta^{t}$ at time $t$ is given by

$$
\Theta^{t}=\bigcup_{\left(X_{i},\left(G_{i}, \alpha_{i}\right)\right) \in N} B_{R_{i}(t)}\left(X_{i}\right),
$$

with $R_{i}(t):=G_{i} \int_{0}^{t} g\left(\tau, \alpha_{i}\right) \mathrm{d} \tau$.

By observing that the causal cone is now given by

$$
\mathscr{C}(t, x):=\left\{(y, \xi, a) \in \mathbb{R}^{d} \times \mathbb{R}_{+} \times \mathbb{R}^{n}: x \in B_{R(t)}(y)\right\}
$$

with $R(t):=\xi \int_{0}^{t} g(\tau, a) \mathrm{d} \tau$, and by proceeding along the same lines of the previous section, it can be proved that Eqs.6, 4 and 7 still hold with

$$
\Lambda(\mathscr{C}(t, x))=\int_{\mathbb{R}_{+} \times \mathbb{R}^{n}} \int_{B_{R(t)}(x)} \lambda(y) \mathrm{d} y Q(\mathrm{~d}(\xi, a)),
$$

and $S_{E}$ is now given by

$S_{E}(t, x)=\int_{\mathbb{R}_{+} \times \mathbb{R}^{n}} \int_{\partial B_{R(t)}(x)} \lambda(y) \mathscr{H}^{d-1}(\mathrm{~d} y) Q(\mathrm{~d}(\xi, a))$,

(provided, of course, that the above integrals exists finite).

Corollary 7 If $\lambda$ is a non-negative harmonic function in the spatial region where the nucleation takes place, and if $G_{0}$ and $\alpha$ are independent with probability distribution $Q_{1}$ and $Q_{2}$, respectively, then the above equations simplify as follows:

$$
\begin{aligned}
V_{E}(t, x) & =\Lambda(\mathscr{C}(t, x)) \\
& =\lambda(x) b_{d} \mathbb{E}\left[G_{0}^{d}\right] \mathbb{E}\left[\left(\int_{0}^{t} g(\tau, \alpha) \mathrm{d} \tau\right)^{d}\right], \\
S_{E}(t, x) & =\lambda(x) d b_{d} \mathbb{E}\left[G_{0}^{d-1}\right] \mathbb{E}\left[\left(\int_{0}^{t} g(\tau, \alpha) \mathrm{d} \tau\right)^{d-1}\right] .
\end{aligned}
$$

Example By the above general results, we can now reobtain as particular cases some known expressions for the mean densities considered in literature in facing certain problems of applicative interest. For instance, in the analytical work by Juul Jensen et al. (2002) and in the computer simulations in Godiksen et al. (2007), the 3D case is considered, with $\lambda(x)=\lambda$ constant and $G(t)$ of the type Eq. 21 with

$$
g(t, \alpha)=(1-\alpha) t^{-\alpha}
$$

and so the radius of the grain at time $t$ with nucleus $X_{i}$ is given by $R_{i}(t)=G_{i} t^{1-\alpha_{i}}$. In particular Godiksen et al. (2007) simulate two cases: in the first one they assume $\alpha=0$ (such a case coincides with the random constant velocity case discussed in the previous section); in the second one they assume deterministic $G_{0} \equiv 1$ and $\alpha$ random with distribution uniform in $[0,1]$ or with distribution $\operatorname{Bet} a(a, b)$. Of course, we can handle and generalise these cases easily; in particular, assuming also $G_{0}$ random and $\lambda$ non-constant harmonic function, Eq. 23 becomes in the $3 D$ case:

$$
V_{E}(t, x)=\frac{4}{3} \pi \lambda(x) \mathbb{E}\left[G_{0}^{3}\right] \mathbb{E}\left[t^{3(1-\alpha)}\right]
$$

so that we get

$$
V_{V}(t, x)=1-\exp \left\{-\lambda(x) \frac{4}{3} \pi \mathbb{E}\left[G_{0}^{3}\right] \int_{0}^{1} t^{3(1-z)} \mathrm{d} z\right\},
$$

when $\alpha \sim U[0,1]$, and

$$
\begin{aligned}
& V_{V}(t, x)=1-\exp \left\{-\lambda(x) \frac{4}{3} \pi \mathbb{E}\left[G_{0}^{3}\right]\right. \\
& \left.\int_{0}^{1} t^{3-3 y} \frac{\Gamma(a+b)}{\Gamma(a) \Gamma(b)} y^{a-1}(1-y)^{b-1} \mathrm{~d} y\right\}
\end{aligned}
$$

when $\alpha \sim \operatorname{Bet} a(a, b)$. (See also Rios and Villa, 2011 for a more exhaustive discussion about this.)

\section{RANDOM CONSTANT POSITION- DEPENDENT VELOCITY}

Assumptions: each grain develops with random velocity constant in time during the reaction, whose probability distribution depends on the spatial position of the corresponding nucleus.

We may model the nucleation process by a position dependent marked Poisson point process with intensity

$$
\Lambda(\mathrm{d}(x, \xi))=\lambda(x) \mathrm{d} x Q(x, \mathrm{~d} \xi)
$$




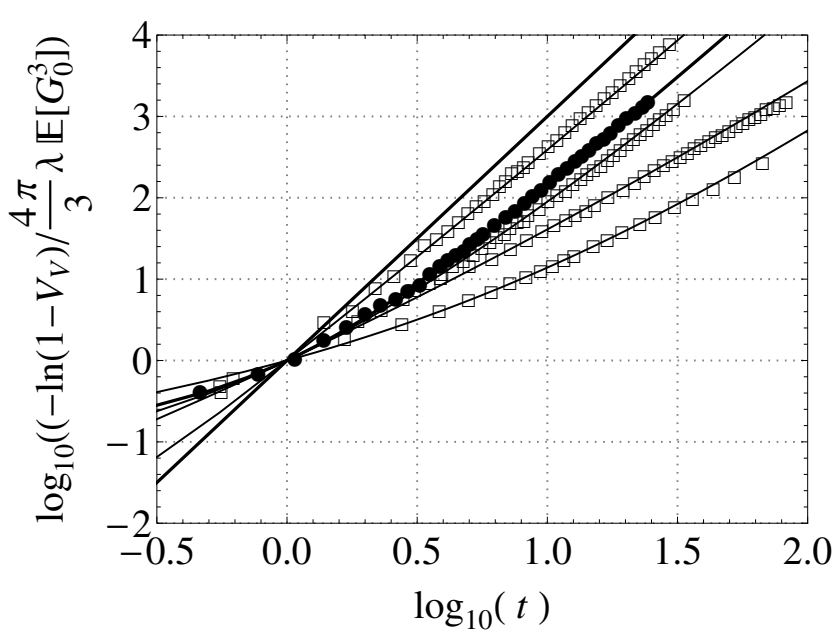

Fig. 2. The straight line without any symbols on it corresponds to the KJMA kinetics for a constant, nonrandom velocity. The symbols correspond to Godiksen et al. simulated data and the solid lines correspond to the theoretical result and were calculated using a uniform distribution, Eq. 24: $(\bullet)$ and using a Beta distribution, Eq. 25: ( $\square$ ), for different values of a and $b$. There is, as expected, excellent agreement between simulation and analytical solution.

Note that now $Q(y, \mathrm{~d} \xi)$ represents the probability distribution of $G$ given that the associated nucleus is born in $y$. Of course, the case discussed in the previous section "Random constant velocity" can be seen now as a particular case. By proceeding along the same lines as before, Eqs. 6, 4 and 7 still hold now with the causal cone given by

$$
\begin{aligned}
\mathscr{C}(t, x): & \left\{(y, \xi) \in \mathbb{R}^{d} \times \mathbb{R}_{+}: x \in B_{\xi t}(y)\right\} \\
& =\left\{(y, \xi) \in \mathbb{R}^{d} \times \mathbb{R}_{+}: \xi \geq \operatorname{dist}(y, x) / t\right\},
\end{aligned}
$$

and so

$$
\begin{aligned}
V_{E}(t, x)= & \Lambda(\mathscr{C}(t, x))= \\
& \int_{\mathbb{R}^{d}}\left(\int_{\operatorname{dist}(y, x) / t}^{\infty} Q(y, \mathrm{~d} \xi)\right) \lambda(y) \mathrm{d} y .
\end{aligned}
$$

As we can see, such a case could be more difficult to handle because the evaluation of the integral above might be quite hard.

Example Let us consider the case $Q(y, \mathrm{~d} \xi):=$ $\frac{1}{y_{1}} \mathbf{1}_{\left[0, y_{1}\right]}(\xi) \mathrm{d} \xi$, i.e., $G(y) \sim U\left[0, y_{1}\right]$; then

$$
\begin{aligned}
& V_{V}(t, x) \stackrel{\text { Eq. }}{=} \\
& 1-\exp \left\{-\int_{\left\{y \in \mathbb{R}^{d}: \frac{\operatorname{dist}(y, x)}{t} \leq y_{1}\right\}} \frac{y_{1}-\operatorname{dist}(y, x) / t}{y_{1}} \lambda(y) \mathrm{d} y\right\} .
\end{aligned}
$$

\section{Particular case: position-dependent deterministic velocity}

Let us assume that each grain grows with constant velocity, depending on the spatial location of the corresponding nucleus, i.e., the velocity $G_{i}$ associated to a grain with nucleus in $X_{i} \in \mathbb{R}^{d}$ is equal to $f\left(X_{i}\right)$, where $f$ is a given function from $\mathbb{R}^{d}$ to $\mathbb{R}_{+}$. It follows that the transformed region $\Theta^{t}$ at time $t$ is the union of balls with random centres of the type:

$$
\Theta^{t}=\bigcup_{X_{i} \in N} B_{f\left(X_{i}\right) t}\left(X_{i}\right),
$$

where $N$ is a Poisson nucleation process in $\mathbb{R}^{d}$ with intensity $\lambda$.

This model can be seen as a particular case of the previous one, with

$$
Q(y, \mathrm{~d} \xi):=\delta_{f(y)}(\xi) \mathrm{d} \xi,
$$

where $\delta_{f(y)}$ is the Dirac delta function in $f(y)$. Thus, by denoting

$$
A(x, t)=\left\{y \in \mathbb{R}^{d}: f(y) t \geq \operatorname{dist}(y, x)\right\},
$$

Eq. 26 simplifies as follows:

$$
V_{E}(t, x)=\int_{A(x, t)} \lambda(y) \mathrm{d} y .
$$

Since the nucleation process is Poissonian we still have $V_{V}(t, x)=1-\exp \left\{-V_{E}(t, x)\right\}$.

Note that, under homogeneous nucleation with $\lambda(x) \equiv \lambda$, we have $V_{V}(t, x)=1-\exp \left\{-\lambda v^{d}(A)\right\}$, so that only the volume of the set $A(x, t)$ has to be evaluated; explicitly, for practical purposes in the $3 \mathrm{D}$ case $A=\left\{\left(y_{1}, y_{2}, y_{3}\right) \in \mathbb{R}^{3}: f(y) t \geq\right.$ $\left.\sqrt{\left(y_{1}-x_{1}\right)^{2}+\left(y_{2}-x_{2}\right)^{2}+\left(y_{3}-x_{3}\right)^{2}}\right\}$.

\section{RANDOM VELOCITY IN THE TIME-DEPENDENT CASE}

In this section we consider the case in which the nucleation takes place randomly both in time and space, and the growth velocity of the grains is random.

In the site-saturated case we modelled the random velocity of a certain grain as a mark of the corresponding nucleus, and so by modelling the nucleation process as a marked Poisson point process. Analogously, we shall add a further mark to the nucleation process $\left\{T_{i}, X_{i}\right\}$, by marking the point process $\left\{T_{i}\right\}_{i}$ in $\mathbb{R}_{+}$of the birth-times of the nuclei with marks representing the spatial location of the nucleus and the velocity of the corresponding grains. 
It is intuitive that arguments and results are similar to those of the previous section; difficulties in the timedependent case might be arise in evaluation of the involved integrals. We shall discuss the three cases of interest studied in the site-saturated case.

We assume that the point process $\left\{T_{i}\right\}_{i}$ describing the birth time of the nuclei is an inhomogeneous Poisson point process in $\mathbb{R}_{+}$with intensity $\lambda(s)$. We also assume independent marking of $\left\{T_{i}\right\}_{i}$, that is the intensity measure of the marked point process is of the type $\Lambda(\mathrm{d}(s, k))=\lambda(s) \mathrm{d} s Q(\mathrm{~d} k)$, where $Q$ is the mark distribution, i.e., a probability measure on the mark space.

\section{RANDOM CONSTANT POSITION- DEPENDENT VELOCITY}

Assumptions: each grain develops with random velocity, constant in time during the reaction. The velocity $G_{i}$ associated to the grain born at time $T_{i}$ at point $X_{i}$ is in general dependent on the spatial location $X_{i}$. We denote by $Q(\mathrm{~d}(y, \xi))$ the joint probability law of $\left(X_{i}, G_{i}\right)$.

Therefore we may consider the spatial location $X_{i}$ and the velocity $G_{i}$ associated to the birth-time $T_{i}$ as marks of $T_{i}$, and model such a birth-and-growth process by a Poisson point process $N=\left\{\left(T_{i},\left(X_{i}, G_{i}\right)\right)\right\}$ in $\mathbb{R}_{+}$with marks in $\mathbb{R}^{d} \times \mathbb{R}_{+}$, having intensity measure

$$
\Lambda(\mathrm{d} s, \mathrm{~d}(y, \xi)=\lambda(s) \mathrm{d} s Q(\mathrm{~d}(y, \xi)) .
$$

Note that $Q(\mathrm{~d}(y, \xi))$ represents the probability that any nucleus is born in the infinitesimal region $\mathrm{d} y$ and the growth velocity of the associated grain is in the infinitesimal interval $\mathrm{d} \xi$. So, by giving a suitable joint probability $Q$ we can easily handle the case of positiondependent velocity.

It follows that the transformed region $\Theta^{t}$ at time $t$ is given by

$$
\Theta^{t}=\bigcup_{\left(T_{i},\left(X_{i}, G_{i}\right)\right) \in N} B_{G_{i}\left(t-T_{i}\right)}\left(X_{i}\right),
$$

so that the causal cone of any point $x \in \mathbb{R}^{d}$ at time $t \in \mathbb{R}_{+}$is now the subset of $\mathbb{R}_{+} \times \mathbb{R}^{d} \times \mathbb{R}_{+}$so defined $\mathscr{C}(t, x)=\left\{(s,(y, \xi)) \in \mathbb{R}_{+} \times \mathbb{R}^{d} \times \mathbb{R}_{+}: y \in B_{\xi(t-s)}(x)\right\}$. Again we have that

$$
\left.V_{V}(t, x)=1-\exp \left\{-V_{E}(t, x)\right)\right\},
$$

with

$$
\begin{aligned}
\left.V_{E}(t, x)\right) & =\Lambda(\mathscr{C}(t, x)) \\
& =\int_{0}^{t} \lambda(s)\left(\int_{\mathbb{R}_{+}} \int_{B_{\xi(t-s)}(x)} Q(\mathrm{~d}(y, \xi))\right) \mathrm{d} s .
\end{aligned}
$$

Remark 8 (Position-independent velocity) In case $G_{i}$ and $X_{i}$ are independent, then the mark distribution factorizes as $Q(\mathrm{~d}(y, \xi))=Q_{X}(\mathrm{~d} x) Q_{G}(\mathrm{~d} \xi)$, having denoted by $Q_{X}$ and $Q_{G}$ the probability distribution of the random location $X$ of the nuclei and of the velocity $G$, respectively, and so the intensity measure $\Lambda$ of the process is of the type $\Lambda(\mathrm{d} s, \mathrm{~d}(y, \xi))=$ $\lambda(s) \mathrm{d} s Q_{X}(\mathrm{~d} y) Q_{G}(\mathrm{~d} \xi)$.

In some applications it is of interest to evaluate the mean volume density $V_{V}$ in the centre of the specimen; note that in the particular case in which the nucleation is homogenous in time (i.e., $\lambda(s) \equiv \lambda>0$ ), the nuclei are uniformly located in a compact window $[-M, M]^{d}$ and $G$ is bounded, say $G \leq K \in \mathbb{R}_{+}$, then by Eq. 27 we get:

$$
V_{E}(t, 0)=\frac{\lambda b_{d} t^{d+1}}{2^{d} M^{d}(d+1)} \mathbb{E}\left[G^{d}\right], \quad \forall t \in[0, M / K] .
$$

Example Under the above assumptions, we get the following explicit expressions for the mean volume density $V_{V}(t, x)$ in some cases of interests for applications:

- if the velocity $G$ and the spatial location $X$ of the nuclei are independent, with $G \sim U(0, K)$ and $X \sim U\left([-M, M]^{d}\right)$, then

$$
Q(\mathrm{~d}(y, \xi))=\frac{1}{2^{d} M^{d} K} \mathbf{1}_{[-M, M]^{d}}(y) \mathbf{1}_{[0, K]}(\xi) \mathrm{d} y \mathrm{~d} \xi,
$$

and so

$$
\begin{aligned}
& V_{V}(t, x)=1-\exp \left\{-\frac{1}{2^{d} M^{d} K} \int_{0}^{t} \lambda(s)\right. \\
& \left.\left(\int_{0}^{K} v^{d}\left(B_{\xi(t-s)}(x) \cap[-M, M]^{d}\right) \mathrm{d} \xi\right) \mathrm{d} s\right\} .
\end{aligned}
$$

If in particular $\lambda(x) \equiv \lambda>0$, then the volume density at the origin is given by

$$
\begin{array}{r}
V_{V}(t, 0)=1-\exp \left\{-\frac{\lambda b_{d} K^{d} t^{d+1}}{2^{d} M^{d}(d+1)^{2}}\right\}, \\
\forall t \in[0, M / K] .
\end{array}
$$

- if the velocity $G$ and the spatial location $X$ of the nuclei are independent, with $X \sim U\left([-M, M]^{d}\right)$ and $G$ discrete random variable with $\mathbb{P}\left(G=G_{i}\right)=$ $p_{i}, p_{i} \in[0,1], \sum_{i} p_{i}=1$, then

$$
Q(\mathrm{~d}(y, \xi))=\frac{1}{2^{d} M^{d}} \mathbf{1}_{[-M, M]^{d}}(y) \mathrm{d} y \sum_{i} p_{i} \delta_{G_{i}}(\xi) \mathrm{d} \xi,
$$


and so

$$
\begin{aligned}
& V_{V}(t, x)=1-\exp \left\{-\frac{1}{2^{d} M^{d}} \int_{0}^{t} \lambda(s)\right. \\
& \left.\left(\sum_{i} v^{d}\left(B_{G_{i}(t-s)}(x) \cap[-M, M]^{d}\right) p_{i}\right) \mathrm{d} s\right\} .
\end{aligned}
$$

If in particular $\lambda(s) \equiv \lambda>0$, and if $\sup _{i} G_{i}=K$, then the volume density at the origin is given by

$$
\begin{aligned}
V_{V}(t, 0) & =1-\exp \left\{-\frac{\lambda b_{d}}{2^{d} M^{d}} \int_{0}^{t} \sum_{i} G_{i}^{d}(t-s)^{d} p_{i} \mathrm{~d} s\right\} \\
& =1-\exp \left\{-\frac{\lambda b_{d} t^{d+1}}{2^{d} M^{d}(d+1)} \mathbb{E}\left[G^{d}\right]\right\}
\end{aligned}
$$

$\forall t \in[0, M / K]$, in accordance with Eq. 28 .

\section{RANDOM TIME-DEPENDENT VELOCITY}

Assumptions: each grain develops with random time-dependent velocity during the reaction of the type Eq. 21.

Thus we can model the nucleation process by a marked Poisson point process $N=\left\{\left(T_{i},\left(X_{i}, G_{i}, \alpha_{i}\right)\right)\right\}$ in $\mathbb{R}_{+}$with marks in $\mathbb{R}^{d} \times \mathbb{R}_{+} \times \mathbb{R}^{n}$, with intensity measure

$$
\Lambda(\mathrm{d}(s, y, \xi, a))=\lambda(s) \mathrm{d} s Q(\mathrm{~d}(y, \xi, a)),
$$

where $Q$ is the joint probability law of $\left(X, G_{0}, \alpha\right)$, and, in general, with $G$ dependent on the spatial location $X$ of the corresponding nucleus. The growing region $\Theta^{t}$ at time $t$ is given by

$$
\Theta^{t}=\bigcup_{\left(T_{i},\left(X_{i}, G_{i}, \alpha_{i}\right)\right) \in N: T_{i} \leq t} B_{R\left(T_{i}, t\right)}\left(X_{i}\right),
$$

with $R_{i}(s, t):=G_{i} \int_{T_{i}}^{t} g\left(\tau, \alpha_{i}\right) \mathrm{d} \tau$. It is easy to see that Eqs 4 and 6 still hold with

$V_{E}(t, x)=\int_{0}^{t} \lambda(s)\left(\int_{B_{R(s, t)}(x) \times \mathbb{R}_{+} \times \mathbb{R}^{n}} Q(\mathrm{~d}(y, \xi, a))\right) \mathrm{d} s$.

Sometimes it is needed to model nucleations such that the birth-time and the spatial location of the nuclei are dependent; a particular case in which they are independent of the growth velocity of the grains can be model by choosing an intensity measure of the type

$$
\Lambda(\mathrm{d}(s, y, \xi, a))=\lambda(s, y) \mathrm{d} s \mathrm{~d} y Q_{G, \alpha}(\mathrm{d}(\xi, a),
$$

where $Q_{G, \alpha}$ is the joint distribution of $G_{0}$ and $\alpha$. By proceeding along the same lines of Theorem 3 and of Theorem 5, it is not difficult to prove that, if $\Lambda$ is of the type 29 with $\lambda$ bounded and continuous, then Eq. 7 holds and

$$
\begin{aligned}
& S_{E}(t, x)=\int_{0}^{t} \int_{\mathbb{R}_{+} \times \mathbb{R}^{n}} \\
& \quad\left(\int_{\partial B_{R(s, t)}(x)} \lambda(s, y) \mathscr{H}^{d-1}(\mathrm{~d} y)\right) \mathrm{d} s Q_{G, \alpha}(\mathrm{d}(\xi, a),
\end{aligned}
$$

where $R(s, t)=\xi \int_{s}^{t} g(\tau, a) \mathrm{d} \tau$. Note that the above assertion follows by proceeding similarly to the proof of Theorem 5, and observing that

$$
\Theta_{\oplus r}^{t}=\bigcup_{\left(T_{i},\left(X_{i}, G_{i}, \alpha_{i}\right)\right) \in \Phi: T_{i} \leq t} B_{R_{i}(s, t)+r}\left(X_{i}\right),
$$

and $\mathscr{D}_{r}$ given now by $\mathscr{D}_{r}:=\left\{(s, y, \xi, a) \in[0, t] \times \mathbb{R}^{d} \times\right.$ $\left.\mathbb{R}_{+} \times \mathbb{R}^{n}: y \in B_{R(s, t)+r}(x)\right\}$. If moreover $\lambda(s, \cdot)$ is harmonic for any $s \in \mathbb{R}_{+}$, then,

$$
\begin{aligned}
& S_{E}(t, x)= \\
& \quad \int_{0}^{t} \lambda(s, x) d b_{d}\left(\int_{\mathbb{R}_{+} \times \mathbb{R}^{n}}(R(s, t))^{d-1} Q_{G, \alpha}(\mathrm{d}(\xi, a))\right) \mathrm{d} s,
\end{aligned}
$$

and

$$
\begin{aligned}
& V_{E}(t, x)= \\
& \quad \int_{0}^{t} \lambda(s, x) b_{d}\left(\int_{\mathbb{R}_{+} \times \mathbb{R}^{n}}(R(s, t))^{d} Q_{G, \alpha}(\mathrm{d}(\xi, a))\right) \mathrm{d} s .
\end{aligned}
$$

Example If the spatial location $X$ of the nuclei, $G_{0}$ and $\alpha$ are independent, with probability distribution $Q_{X}, Q_{G}$ and $Q_{\alpha}$, respectively, then

$$
Q(\mathrm{~d}(y, \xi, a))=Q_{X}(\mathrm{~d} y) Q_{G}(\mathrm{~d} \xi) Q_{\alpha}(\mathrm{d} a)
$$

and so

$$
\begin{aligned}
& V_{V}(t, x)=1-\exp \left\{-\int_{0}^{t} \lambda(s)\right. \\
& \left.\left(\int_{\mathbb{R}_{+} \times \mathbb{R}_{+}} Q_{X}\left(B_{R(s, t)}(x)\right) Q_{G}(\mathrm{~d} \xi) Q_{\alpha}(\mathrm{d} a)\right) \mathrm{d} s\right\} .
\end{aligned}
$$

Let us notice that if $X \sim U\left([-M, M]^{d}\right)$, then

$$
Q_{X}\left(B_{R(s, t)}(x)\right)=\frac{1}{2^{d} M^{d}} v^{d}\left(B_{R(s, t)}(x) \cap[-M, M]^{d}\right),
$$

it follows that, in the case $g(t, \alpha)=(1-\alpha) t^{-\alpha}$, for $x=0$ and $t$ sufficiently small:

$$
\begin{array}{r}
v^{d}\left(B_{R(s, t)}(0) \cap[-M, M]^{d}\right)=v^{d}\left(B_{R(s, t)}(0)\right) \\
=b_{d} \xi^{d}\left(t^{1-a}-s^{1-a}\right)^{d} .
\end{array}
$$

In the next example, we further specify the previous one, by providing some explicit expressions in a few particular cases of interest for applications. 


\section{Example}

- Let us consider the case in which the nucleation process takes place homogeneously in time, uniformly in a window $[-M, M]^{d}$; we also assume that the velocity of the grains does not depend on the birth-time and spatial position of the corresponding nucleus, and it is of the type $G(t)=$ $G_{0}(1-\alpha) t^{-\alpha}$, with $\alpha$ and $G_{0}$ independent of each other and uniformly distributed in $[0,1]$ and in $[0, K]$, respectively. Therefore

$$
\begin{aligned}
& \Lambda(\mathrm{d}(s, y, \xi, a))= \\
& \quad \frac{\lambda}{2^{d} M^{d} K} \mathbf{1}_{[-M, M]^{d}}(y) \mathbf{1}_{[0, K]}(\xi) \mathbf{1}_{[0,1]}(a) \mathrm{d} s \mathrm{~d} y \mathrm{~d} \xi \mathrm{d} a,
\end{aligned}
$$

and for $x=0$ and $t$ sufficiently small we have

$$
\begin{gathered}
V_{V}(t, 0)=1-\exp \left\{-\frac{\lambda}{2^{d} M^{d} K} \int_{0}^{t}\left(\int_{0}^{1}\right.\right. \\
\left.\left.\left(\int_{0}^{K} v^{d}\left(B_{R(s, t)}(0) \cap[-M, M]^{d}\right) \mathrm{d} \xi\right) \mathrm{d} a\right) \mathrm{~d} s\right\} \\
\stackrel{\mathrm{Eq} .30}{=} 1-\exp \left\{-\frac{\lambda b_{d} K^{d}}{2^{d} M^{d}(d+1)}\right. \\
\left.\int_{0}^{1} t^{(1-a) d+1} \sum_{j=0}^{d}\left(\begin{array}{l}
d \\
j
\end{array}\right) \frac{(-1)^{j}}{(1-a)(d-j)+1} \mathrm{~d} a\right\} .
\end{gathered}
$$

- It is clear that some cases of interest in applications (e.g., $G_{0}$ or $\alpha$ deterministic), follow now as particular cases. Let us consider the very simple case in which nucleation takes place homogeneously both in time and space, and $G(t)=$ $G_{0}(1-\alpha) t^{-\alpha}$ with $G_{0}$ and $\alpha$ independent each other. Denoted by $\lambda$ the mean number of nuclei per unit of time and unit of volume, it follows that

$V_{V}(t, x)=1-\exp \left\{-\lambda b_{d} \mathbb{E}\left[G_{0}^{d}\right] \int_{0}^{t}\left(t^{1-\alpha}-s^{1-\alpha}\right)^{d} \mathrm{~d} s\right\}$,

if $G_{0}$ is random and $\alpha$ is deterministic, whereas

$V_{V}(t, x)=1-\exp \left\{-\lambda b_{d} G_{0}^{d} \int_{0}^{t} \mathbb{E}\left[\left(t^{1-\alpha}-s^{1-\alpha}\right)^{d}\right] \mathrm{d} s\right\}$,

if $G_{0}$ is deterministic and $\alpha$ is random.

\section{SUMMARY AND CONCLUSIONS}

- General expressions were derived for the mean volume density and the mean surface density of birth-and-growth processes with Poisson nucleation process and spherical grains with distinct random velocities, modelling recrystallization processes in materials science, both in case of site-saturation and in case of time-dependent nucleation. To this aim, tools from Stochastic Geometry and recent results from Geometric Measure Theory (namely in the proof of Theorem 5), together with the notion of causal cone have been used.

The general case of random growth velocity of the grains with distribution dependent on the spatial location of the corresponding nucleus has been also considered.

Some particular cases of practical relevance in applications have been discussed as examples throughout the paper, also in connection with previous literature.

- Specifically with regard to materials science, these results may be used to model and/or assess the importance of a random velocity distribution in transformations kinetics, as opposed to assume a non-random velocity. It is particularly useful in the cases where the velocity is known to be different for each grain such as in recrystallization as experimentally demonstrated by Juul Jensen and coworkers.

- This paper provides the mathematical basis for the development of more general expressions to be used in practical applications. Although the motivation was recrystallization the expressions derived here may be applied and extended to nucleation and growth reactions in general.

\section{ACKNOWLEDGEMENTS}

P.R.R. is grateful to the Conselho Nacional de Desenvolvimento Científico e Tecnolgico, CNPq, and to the Fundação de Amparo à Pesquisa do Estado do Rio de Janeiro, FAPERJ, for financial support. Both authors are grateful to the anonymous referees for their careful reading of the paper and suggestions.

\section{REFERENCES}

Aquilano D, Capasso V, Micheletti A, Patti S, Pizzocchero L, Rubbo M (2009). A birth and growth model for kinetic-driven crystallization processes. I. Modeling. Nonlinear Anal Real World Appl 10:71-92.

Avrami MJ (1939). Kinetics of phase change I. General theory. J Chem Phys 7:1103-12.

Avrami MJ (1940). Kinetics of phase change. II. Transformation-time relations for random distribution of nuclei. J Chem Phys 8:212-24.

Avrami MJ (1941). Granulation phase change, and microstructure kinetics of phase change. III. J Chem Phys 9:177-84. 
Baddeley A, Bárány I, Schneider R (2007). Stochastic geometry. Lect Notes Math 1892. Berlin: Springer.

Burger M, Capasso V, Salani C (2002). Modelling multidimensional crystallization of polymers in interaction with heat transfer. Nonlinear Anal Real World Appl 3:139-60.

Capasso V, Burger M, Micheletti A, Salani C (2003). Mathematical models for polymer crystallization processes. In: Capasso V, ed. Mathematical modelling for polymer processing. Math Industr 2:167-242. Berlin: Springer.

Capasso V, Villa E (2007a). On the evolution equations of mean geometric densities for a class of space and time inhomogeneous stochastic birth-and-growth processes. In: Baddeley A, Bárány I, Schneider R, eds. Stochastic Geometry. Lect Notes Math 1892:267-81. Berlin: Springer.

Capasso V, Villa E (2007b). On mean densities of inhomogeneous geometric processes arising in material science and medicine. Image Anal Stereol 26:23-36.

Fallert H (1996). Quermaßdichten für Punktprozesse konvexer Körper und Boolesche Modelle. Math Nachr 181:165-84.

Fanfoni M, Tomellini M (2005). Film growth viewed as stochastic dot processes. J Phys Condens Matter 17:R571.

Godiksen RBN, Schmidt S, Juul Jensen D (2007). Effects of distributions of growth rates on recrystallization kinetics and microstructure. Scr Mater 57:345-8.

Juul Jensen D, Godiksen RBN (2008). Neutron and synchrotron X-ray studies of recrystallization kinetics. Metal Mater Trans A 39:3065-69.

Juul Jensen D, Lauridsen EM, Vandermeer RA (2002). Insitu determination of grain boundary migration during recrystallization. In: Ankem A et al., eds. Science and technology of interfaces. Warrendale, PA: TMS, 361-74

Johnson WA, Mehl RF (1939). Reaction kinetics in process of nucleation and growth. Trans AIME 135:416-42.

Jun S, Bechhoefer J (2005). Nucleation and growth in one dimension. II. Application to DNA replication kinetics. Phys Rev E 71:011909.

Kolmogorov AN (1937). On the statistical theory of the crystallization of metals. Bull Acad Sci USSR Math Ser 1:355-9.

Kondo T, Sakuma T, Rios PR (1998). Application of microstructural path analysis to abnormal grain growth of $\mathrm{BaTiO}_{3}$ with an excess $\mathrm{TiO}_{2}$. Scr Mater 39:1713-7.

Lauridsen EM, Poulsen HF, Nielsen SF, Juul Jensen D (2003). Recrystallization kinetics of individual bulk grains in $90 \%$ cold-rolled aluminium. Acta Mater
51:4423-35.

Matheron G (1975). Random sets and integral geometry. New York: John Wiley \& Sons

Ramos RA, Rikvold PA, Novotny MA (1999). Test of the Kolmogorov-Johnson-Mehl-Avrami picture of metastable decay in a model with microscopic dynamics. Phys Rev B 59:9053-69.

Rios PR, Guimaraes JRC (2007). Microstructural path analysis of athermal martensite. Scr Mater 57:1105-8.

Rios PR, Guimaraes JRC (2008). Formal analysis of isothermal martensite spread. Mat Res 11:103-8.

Rios PR, Villa E (2009). Transformation kinetics for inhomogeneous nucleation. Acta Mater 57:1199-208.

Rios PR, Villa E (2011) An analytical approach to the effect of a distribution of growth velocities on recrystallization kinetics. Scripta Mater 65:938-41.

Schneider R, Weil W (2008). Stochastic and integral geometry. Berlin, Heidelberg: Springer.

Starink MJ (2004). Analysis of aluminium based alloys by calorimetry: quantitative analysis of reactions and reaction kinetics. Int Mater Rev 49:191-226.

Stoyan D, Kendall WS, Mecke J (1995). Stochastic geometry and its application. Chichester: Wiley.

Tomellini M, Fanfoni M (2008). Impingement factor in the case of phase transformations governed by spatially correlated nucleation. Phys Rev B 78:014206.

Vandermeer RA, Juul Jensen D (2001). Microstructural path and temperature dependence of recrystallization in commercial aluminum. Acta Mater 49:2083-94.

Vandermeer RA, Masumura RA, Rath BB (1991). Microstructural paths of shape-preserved nucleation and growth transformations. Acta Metall Mater 39:383-9.

Villa E (2008). A note on mean volume and surface densities for a class of birth-and-growth stochastic processes. Int J Contemp Math Sci 3:1141-55.

Villa E (2010a). Mean densities and spherical contact distribution function of inhomogeneous Boolean models. Stoch Anal Appl 28:480-504.

Villa E (2010b). On the specific area of inhomogeneous Boolean models. Existence results and applications. Image Anal Stereol 29:111-9.

Villa E, Rios PR (2009). Transformation kinetics for nuclei clusters. Acta Mater 57:3714-24.

Villa E, Rios PR (2010). Transformation kinetics for surface and bulk nucleation. Acta Mater 58:2752-68.

Villa E, Rios PR (2011). Transformation kinetics for nucleation on random planes and lines. Image Anal Stereol 30:153-65. 